\title{
The Cellular Mechanisms of Learning in Aplysia: Of Blind Men and Elephants
}

\author{
DAVID L. GLANZMAN* \\ Department of Physiological Science, UCLA College, and Department of Neurobiology and the Brain \\ Research Institute, David Geffen School of Medicine at UCLA, Los Angeles, California 90095
}

\begin{abstract}
Until recently, investigations of the neurobiological substrates of simple forms of learning and memory in the marine snail Aplysia have focused mostly on plastic changes that occur within the presynaptic sensory neurons. Here, I summarize the results of recent studies that indicate that exclusively presynaptic processes cannot account for simple forms of learning in Aplysia. In particular, I present evidence that postsynaptic mechanisms play a far more important role in nonassociative learning in Aplysia than has been appreciated before now. Moreover, I describe recent data that suggests the intriguing hypothesis that the persistent, learning-induced changes in Aplysia sensory neurons might depend critically on postsynaptic signals for their induction. Finally, I discuss the potential applicability of this hypothesis to learning-related synaptic plasticity in the mammalian brain.
\end{abstract}

\section{Introduction}

A review of the literature on learning-related synaptic plasticity from the last two decades brings to mind the fable of the blind men and the elephant. As re-told in John Godfrey Saxe's poem,

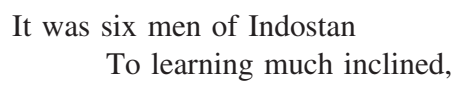

(Though all of them were blind),

Received 17 February 2006; accepted 13 April 2006.

* To whom correspondence should be addressed, at Gonda (Goldschmied) Neuroscience and Genetics Research Center, UCLA, 695 Young Drive South, Box 951761, Los Angeles, CA 90095-1761. E-mail: dglanzman@physci.ucla.edu

Abbreviations: EPSP, excitatory postsynaptic potential; LLH, long-lasting habituation; LTD, long-term depression; LTH, long-term habituation; LTP, long-term potentiation; STH, short-term habituation.
That each by observation Might satisfy his mind.

In the fable each of the blind men feels a single part of the elephant and comes to a very different conclusion about the nature of the animal. While the man who holds the elephant's trunk concludes that the elephant is "very like a snake," another, who feels only the elephant's ear, claims that the beast resembles a fan. Yet a third blind man, who touches one of the tusks, argues that the animal is like a spear. As the poem ends,

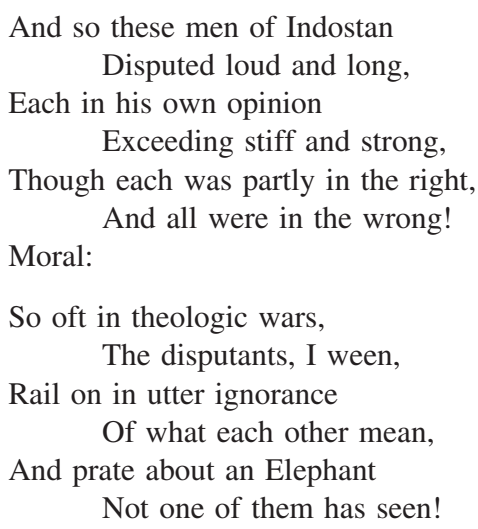

The intensity of the scientific debates concerning one prominent form of synaptic plasticity, long-term potentiation (LTP) of synapses in the mammalian hippocampus (Malenka and Nicoll, 1999), has probably seemed at times reminiscent of "theologic wars" to those outside the field. The ongoing dispute over the site, whether pre- or postsynaptic, of LTP expression-a term that refers to the cellular modifications that underlie the persistence of the synaptic enhancement-has been especially ferocious. In the past several years, however, it has become increasingly evident that expression of LTP of synapses in the CA1 region of the hippocampus almost certainly involves both postsynaptic 
changes-particularly modulation of the trafficking of $\alpha$-amino-3-hydroxy-5-methyl-4-isoxazolepropionic acid (AMPA)-type glutamate receptors (Nicoll, 2003) - and presynaptic changes (Antonova et al., 2001; Choi et al., 2003; Zakharenko et al., 2003; Powell, 2006). This emerging consensus raises the intriguing question of whether any form of persistent (lasting for more than $\approx 10 \mathrm{~min}$ ), learning-induced synaptic plasticity can result from changes to only one side of a synapse.

Many neuroscientists, if this question were posed to them, would be likely to respond affirmatively; moreover, they would probably cite the research on learning and memory in Aplysia (Byrne and Kandel, 1996; Kandel, 2001) as providing the strongest evidence for "one-sided" synaptic plasticity. As described in most introductory textbooks of neuroscience, this mollusc exhibits a simple withdrawal reflex that can exhibit several simple forms of learning, including habituation, sensitization, and classical conditioning. Until relatively recently, each of these forms of learning has been ascribed, predominately or exclusively, to changes in the sensory neurons that mediate the withdrawal reflex. Thus, habituation of the reflex has been thought to be due to homosynaptic (presynaptic) depression (Castellucci and Kandel, 1974; Kandel et al., 1975; Armitage and Siegelbaum, 1998), sensitization to presynaptic facilitation (Kandel et al., 1975; Byrne and Kandel, 1996; Kandel, 2001), and classical conditioning to activity-dependent presynaptic facilitation (Hawkins et al., 1983; Walters and Byrne, 1983; Carew et al., 1984). Within the last decade, however, this tidy view of the cell biology of learning in Aplysia has undergone significant revision. It is now generally acknowledged, for example, that classical conditioning of the withdrawal reflex requires Hebbian, $N$-methyl-D-aspartate (NMDA) receptor-dependent LTP of the sensorimotor synapse (Lin and Glanzman, 1994a, b; Murphy and Glanzman, 1997, 1999; Antonov et al., 2003), as well as a rise in intracellular $\mathrm{Ca}^{2+}$ within the postsynaptic motor neuron (Murphy and Glanzman, 1996; Bao et al., 1998; Antonov et al., 2003; for review, see Roberts and Glanzman, 2003). Moreover, recent evidence indicates that not only associative learning, but also nonassociative learning, in Aplysia depends critically on postsynaptic changes. Below I summarize data from my laboratory, as well as from other laboratories, that implicate postsynaptic mechanisms in habituation and sensitization.

\section{The Role of Postsynaptic Glutamate Receptors in Behavioral Habituation}

Repeated, innocuous tactile stimulation of the animal's siphon produces habituation of the defensive withdrawal reflex in Aplysia. Both short- and long-term forms of habituation have been demonstrated (Pinsker et al., 1970; Carew et al., 1972; Carew and Kandel, 1973). Using a reduced preparation, Youssef Ezzeddine and I (Ezzeddine and Glanz- man, 2003) tested whether persistent habituation of the gill-withdrawal reflex depends on activation of glutamate receptors within the abdominal ganglion. (Ezzeddine and I originally termed this form of habituation long-lasting habituation, or LLH. Because we have recently found that the persistent habituation requires gene expression [see below], however, we now refer to the learning as long-term habituation [LTH].) In our experiments, gill withdrawal was stimulated by weak electrical stimulation of the siphon. Electrodes were implanted into both sides of the siphon; stimulation of one side of the siphon was used for habituation training, while the other side of the siphon served as the control (untrained) side. The strength of the withdrawal reflex in response to siphon stimulation was measured with a force transducer attached to the gill. The artery leading to the abdominal ganglion was cannulated so that drugs could be delivered directly to this ganglion, which contains those sensory and motor neurons that mediate the siphon-elicited gill withdrawal.

We found that LTH of gill withdrawal depends on both protein synthesis and gene expression, because it is blocked by infusion into the abdominal ganglion of either anisomycin (Ezzeddine and Glanzman, 2003) or actinomycin-D (Ezzeddine et al., 2004). Furthermore, LTH requires activation of the protein phosphatases 1 and 2A (PP1 and PP2A) (Ezzeddine and Glanzman, 2003) and calcineurin (Ezzeddine et al., 2004). Surprisingly-and in contrast to the conclusions of previous cellular analyses of short- and long-term habituation in Aplysia (Castellucci et al., 1970; Castellucci and Kandel, 1974; Bailey and Chen, 1983, 1988a, b) —we discovered that LTH depends on activation of glutamate receptors. In particular, LTH was blocked when habituation training was carried out in the presence of either the NMDA receptor antagonist APV or the AMPA receptor antagonist DNQX (Ezzeddine and Glanzman, 2003). (Note that testing in the experiments was performed in normal artificial seawater.) The latter result is particularly interesting, because it distinguishes the mechanism of LTH from that of classic homosynaptic depression. As shown by Armitage and Siegelbaum (1998), short-term homosynaptic depression of the in vitro sensorimotor synapse proceeds normally with repeated activation of the sensory neuron despite a blockade of (presumably) postsynaptic glutamate receptors by DNQX sufficient to eliminate the sensorimotor excitatory postsynaptic potential (EPSP). Therefore, induction of homosynaptic depression does not require activation of postsynaptic AMPA-type receptors. Taken together, the data of Armitage and Siegelbaum (1998) and those of Ezzeddine and Glanzman (2003) demonstrate that presynaptic depression of transmitter release at sensorimotor synapses, although a likely cellular mechanism for short-term habituation (STH), cannot account for LTH.

There is good experimental evidence that depression at central sensorimotor synapses makes a significant contribu- 
tion to behavioral habituation in Aplysia (Cohen et al., 1997; Frost et al., 1997). Heretofore, this depression has been ascribed to presynaptic processes (Castellucci et al., 1970; Castellucci and Kandel, 1974). But if, as our data suggest, the induction of synaptic depression during LTH does not occur presynaptically, what mechanism might underlie the habituation-induced depression of sensorimotor synapses? A potential candidate is a mechanism resembling long-term depression (LTD) of synapses in the mammalian brain (Malenka and Bear, 2004). One form of LTD, prominent at synapses in the CA1 region of the hippocampus and in the cortex, is induced by repeated, low-frequency homosynaptic stimulation. Induction of this form of LTD requires activation of NMDA receptors (Dudek and Bear, 1992; Mulkey and Malenka, 1992), and involves activity of postsynaptic PP1 and calcineurin (Mulkey et al., 1993, 1994; Kirkwood and Bear, 1994). Induction of LTH in Aplysia similarly requires NMDA receptor activation, as well as PP1/PP2A and calcineurin (Ezzeddine and Glanzman, 2003; Ezzeddine et al., 2004). But we do not yet know whether the critical signaling cascades in which these molecules participate occur within the motor neurons. A form of LTD-like plasticity has been previously demonstrated for the in vitro sensorimotor synapse (Lin and Glanzman, 1996). Similar to LTD of hippocampal CA1 synapses, homosynaptic LTD of the sensorimotor synapse can be induced by prolonged low-frequency stimulation ( $15 \mathrm{~min}$ at 2 $\mathrm{Hz}$ ), and requires elevation of postsynaptic $\mathrm{Ca}^{2+}$. It remains to be determined, however, whether LTD of sensorimotor synapses in the abdominal ganglion of Aplysia contributes to LTH.

Previous studies have indicated that LTH in Aplysia is expressed, in part, presynaptically. Bailey and Chen $(1983,1988 \mathrm{a})$ have shown that behavioral training that induces LTH of siphon withdrawal produces a significant decrease in the number, size, and presynaptic vesicle complement of active zones associated with siphon sensory neurons, as well as fewer presynaptic varicosities on the branches of siphon sensory neurons. But postsynaptic changes might also play a role in LTH expression. Support for postsynaptic mechanisms of expression in LTH has come from recent studies of Caenorhabditis elegans. This worm reverses its direction of swimming in response to a mechanical tap delivered to the side of the petri dish in which it lives. The reversal (or withdrawal) response habituates with repeated taps (Rose and Rankin, 2001). A distributed training regimen of several spaced blocks of taps produces LTH (lasting $\geq 24 \mathrm{~h}$ ) of the worm's withdrawal response. Rose and colleagues (2003) have recently shown that LTH in C. elegans, like LTH in Aplysia, depends on activation of glutamate receptors: mutant worms that lack AMPA-type receptors ( $g l r-1$ mutants) exhibit normal STH, but impaired LTH. To examine the role of modulation of postsynaptic glutamate receptors in the expression of LTH, Rose et al. (2003) imaged $g l r-1$ receptors in mutant worms in which the receptors were tagged with green fluorescent protein (GLR-1::GFP worms). Using confocal imaging of GFP expression in the ventral nerve cord of the worm, they found that distributed training resulted in significantly smaller clusters of glutamate receptors. This result suggests that distributed training results in down-regulation of postsynaptic AMPA-type receptors in neurons of the worm's ventral cord, possibly through receptor endocytosis. A similar down-regulation of the surface expression of postsynaptic AMPA receptors is believed to mediate the expression of LTD in the mammalian brain (Malenka and Bear, 2004). Interestingly, presynaptic changes did not appear to play a role in the expression of LTH in C. elegans. Rose et al. (2003) imaged synaptobrevin, a presynaptic vesicle protein, in worms in which synaptobrevin was expressed with GFP; they observed no effect of LTH-inducing training on the amount of synaptobrevin fluorescence in the ventral cords. The results from this study indicate that, whereas presynaptic mechanisms may mediate the induction and expression of STH in C. elegans (see Rankin and Wicks, 2000), LTH appears to depend on activation of AMPA receptors for its induction, and on modulation of the trafficking of AMPA receptors for its expression. Although the finding that LTH in C. elegans does not involve presynaptic mechanisms of expression appears to contrast with the findings of Bailey and Chen (1983, 1988a), it should be pointed out that the animals in the Aplysia studies were subjected to 4-10 days of habituation training; by comparison, the worms received only 1 day of training. Possibly, more extensive behavioral training was necessary to engage the significant presynaptic morphological changes observed by Bailey and Chen.

Whereas our (Ezzeddine and Glanzman, 2003) results suggest that presynaptic mechanisms may be sufficient for STH in Aplysia, a recent study by Antzoulatos et al. (2003) indicates that even very brief forms of synaptic depression may engage postsynaptic processes. These investigators examined the responses of sensorimotor synapses in the central nervous system (CNS) to three presynaptic stimuli delivered at a high frequency $(10 \mathrm{~Hz})$. This stimulation produced homosynaptic depression of the sensorimotor EPSP, as indicated by a reduction in the size of the second and third EPSPs relative to that of the first. When the high-frequency stimulation was delivered in the presence of cyclothiazide, a drug that reduces glutamate receptor desensitization, the depression of the second and third EPSPs was reduced. This result indicates that, at high rates of stimulation, postsynaptic receptor desensitization contributes to depression of the sensorimotor synapse. Whether receptor desensitization plays a role in habituation (or other forms of learning) in Aplysia, however, remains to be determined. 


\section{Postsynaptic Mechanisms of Behavioral Sensitization in Aplysia}

Noxious stimuli, such as strong electrical shocks applied to the animal's skin, produce sensitization/dishabituation of the defensive withdrawal reflex of Aplysia. (Note that, whereas sensitization and dishabituation may engage somewhat distinct cellular mechanisms in Aplysia - [see Hochner et al., 1986; Marcus et al., 1988; Rankin and Carew, 1988; Hawkins et al., 1998] —I will use the term sensitization for both forms of learning, except in those instances where I wish to explicitly refer to dishabituation.) Sensitizing stimuli cause the release of the endogenous monoamine serotonin (5-HT) within the CNS of Aplysia (Glanzman et al., 1989; Mackey et al., 1989; Marinesco and Carew, 2002); and 5-HT facilitates the synaptic connections between central sensory and motor neurons (Brunelli et al., 1976). It has long been believed that 5-HT-induced facilitation of the sensorimotor synapse is mediated by exclusively presynaptic mechanisms (see Byrne and Kandel, 1996, for review). But we have recently found that sensitization-related facilitation of the sensorimotor synapse involves postsynaptic mechanisms, particularly modulation of AMPA receptor trafficking.

The initial evidence that processes within the motor neuron might contribute to 5-HT-induced synaptic facilitation in Aplysia came from experiments performed on isolated siphon motor neurons in cell culture by Randy Chitwood, Quan Li, and myself (Chitwood et al., 2001). We asked whether 5-HT could facilitate the response of the motor neuron to brief applications (puffs) of glutamate, the transmitter used by Aplysia sensory neurons (Dale and Kandel, 1993; Levenson et al., 2000; Chin et al., 2002; but see Trudeau and Castellucci, 1993a). We found that a single, 10-min application of 5-HT causes significant enhancement of the glutamate response evoked in the motor neurons. (Note that Aplysia motor neurons in culture do not form autapses.) The facilitation has a relatively slow onset (5-10 $\min$ ), but persists for $\geq 40 \mathrm{~min}$ after washout of 5-HT. (In more recent experiments we have found that this facilitation can last for as long as $2 \mathrm{~h}$ [G. J. Villareal and D. L. Glanzman, unpubl. data].) The facilitation of the glutamate response depends on both elevated intracellular $\mathrm{Ca}^{2+}$ and G-protein activation, but not on activation of NMDA receptors. Importantly, prior injection of the exocytotic inhibitor botulinum toxin blocked the facilitation. This result indicates that exocytosis of some molecule is necessary for the enhancement of the glutamate response. An important clue to the mechanism of 5-HT's action can be found in our discovery that application of the glutamate receptor antagonist DNQX, although it produced only modest inhibition of the baseline (nonfacilitated) glutamate response in the isolated motor neuron, eliminated the facilitation of the response normally produced by 5 -HT. This suggests that
5-HT might modulate the functional expression of AMPAtype receptors in the motor neuron. Possibly, 5-HT treatment causes the increased surface expression of AMPA receptors in the motor neuron via exocytosis.

To test whether up-regulation of AMPA receptor function plays any role in synaptic facilitation, Quan $\mathrm{Li}$, Adam Roberts, and I ( $\mathrm{Li}$ et al., 2005) turned to experiments on sensorimotor cocultures. We found that the prior injection of BAPTA, a rapid chelator of intracellular $\mathrm{Ca}^{2+}$, into the postsynaptic motor neuron significantly disrupted facilitation of the in vitro synapse due to a 10-min application of 5-HT. Interestingly, chelating postsynaptic $\mathrm{Ca}^{2+}$ did not affect early synaptic facilitation-facilitation lasting for $\approx 5$ min after the start of the 5-HT treatment. However, the presence of BAPTA in the motor neuron significantly degraded the synaptic enhancement at later times. We obtained similar results when we injected heparin, an inhibitor of inositol-1,4,5-triphosphate $\left(\mathrm{IP}_{3}\right)$ receptors, dantrolene, an inhibitor of ryanodine receptors (RyRs), or botulinum toxin into the postsynaptic neuron prior to the application of 5-HT. Taken together, our results indicate that serotonin causes a release of $\mathrm{Ca}^{2+}$ from intracellular stores mediated by both $\mathrm{IP}_{3}$ receptors and RyRs; this release from intracellular stores causes a rise in postsynaptic intracellular $\mathrm{Ca}^{2+}$ which, in turn, drives an exocytotic process required for persistent synaptic facilitation. Early facilitation of the sensorimotor synapse appears not to depend critically on these postsynaptic processes; presumably, then, early synaptic facilitation - which we define as the period of facilitation during 5-10 min after the onset of 5-HT-is mediated predominately by presynaptic processes (Byrne and Kandel, 1996).

To evaluate the potential role played by changes in motor neurons in sensitization-induced facilitation of sensorimotor synapses, Li, Roberts, and I made use of a reduced preparation, which comprised the CNS of Aplysia, together with the pedal (P9) nerves that innervate the animal's tail. We recorded intracellularly from a siphon sensory neuron and one of its postsynaptic siphon motor neurons. We elicited action potentials from the sensory neuron once per $2 \mathrm{~min}$ and recorded the resulting EPSPs from the motor neuron. Extracellular electrical stimulation of the pedal nerves was used to facilitate the sensorimotor EPSP. This facilitation was disrupted by chelating postsynaptic intracellular $\mathrm{Ca}^{2+}$ and by inhibiting postsynaptic $\mathrm{IP}_{3}$ receptors, as was the case for facilitation of the in vitro synapse. Taking advantage of the fact that distinct AMPA and NMDA receptor-mediated components of the sensorimotor EPSP can be isolated by means of the appropriate pharmacological agents (Glanzman, 1994; Antonov et al., 2003), we asked whether pedal nerve shock caused differential facilitation of the different glutamatergic receptor components of the EPSP. We found that after nerve shock there was significantly greater enhancement of the AMPA receptor-mediated component of the EPSP than of the NMDA receptor-mediated component. 
This result is inconsistent with the idea that sensitizationinduced facilitation of the sensorimotor synapse is mediated primarily by enhanced presynaptic release; further, it supports the involvement of AMPA receptor trafficking in sensitization.

Finally, to examine whether AMPA receptor trafficking is involved in actual learning in Aplysia, we used a semiintact preparation. This preparation comprised the CNS, together with the siphon and a portion of the tail, as well as the peripheral nerves that connect these organs to the CNS. The siphon was split; one half was implanted with stimulating electrodes, and the other half was attached to a force transducer. Weak electrical shocks were applied to the siphon via the implanted electrodes, and the resulting contraction of the other half of the siphon was measured with the force transducer. Stimulating electrodes were also implanted in the tail; these were used to deliver strong shocks to this organ. Before the onset of each experiment, two motor neurons, whose intracellular activation produced contraction of the siphon, were identified in the abdominal ganglion. These neurons were loaded with either botulinum toxin or control solution. Subsequently, the siphon was weakly stimulated once per $5 \mathrm{~min}$, which produced habituation of the siphon-withdrawal reflex. After six trials the tail was shocked. Tail shocks produced significant dishabituation in control preparations, but not in preparations in which botulinum toxin had been injected into the motor neurons. This finding indicates that postsynaptic exocytosis, which may mediate insertion of additional AMPA receptors into the postsynaptic membrane, plays a critical role in behavioral dishabituation.

The results described above are reminiscent of those from studies of NMDA receptor-dependent LTP of synapses in the mammalian hippocampus. Work by several investigators has shown that the induction of this form of LTP triggers modulation of postsynaptic AMPA receptor trafficking via activation of calcium/calmodulin-dependent protein kinase II (CaMKII) (Malinow and Malenka, 2002). Furthermore, some evidence suggests that during LTP additional AMPA receptors become incorporated into the postsynaptic membrane through exocytosis (Lledo et al., 1998). Whether modulation of AMPA receptor trafficking in the hippocampus plays a critical role in mammalian learning is not known at present. However, in an experimental tour de force, Malinow and colleagues recently reported that disrupting the synaptic incorporation of new AMPA receptor in neurons in the lateral amygdala-by expressing recombinant proteins in the neurons that block the incorporation of the native AMPA receptors into synapses-impairs fear conditioning in rats (Rumpel et al., 2005). Thus, modulation of AMPA receptor trafficking appears to be a phylogenetically conserved mechanism of learning and memory, one common to both nonassociative and associative memory.

\section{Coordination of Pre- and Postsynaptic Plasticity: A Hypothesis}

The recent work on nonassociative learning in Aplysia suggests that postsynaptic processes play critical roles in both habituation and sensitization. What, then, is the relationship between the presynaptic and postsynaptic mechanisms of memory in Aplysia? Do they operate independently or, rather, are they interdependent? The data from the study by Chitwood et al. (2001), which involved cultures of isolated motor neurons, prove that at least some of the postsynaptic processes that underlie sensitization-related synaptic facilitation do not require any contribution from the sensory neuron. Furthermore, it seems likely from the data of Li et al. (2005) that short-lived facilitation of the sensorimotor synapse can be mediated by exclusively presynaptic processes. It is unclear, however, whether sensory neuron-autonomous processes are sufficient to support persistent facilitation. Initial evidence that a type of learninginduced change in the sensory neuron depends on a postsynaptic signal came from a study by Glanzman et al. (1990). This study examined morphological changes in sensory neurons of sensorimotor cocultures due to repeated applications of 5-HT, a treatment that produces long-term ( $\geq$ 24 h) synaptic facilitation (Montarolo et al., 1986). The 5-HT treatment, as well as facilitating the in vitro synapse, caused the growth of new branches and varicosities on the sensory neurons. However, 5-HT did not produce morphological changes in isolated sensory neurons in culture. This result implies that a retrograde signal is necessary for the long-term presynaptic morphological changes. Recently, Diancai Cai, Quan Li, and I have discovered that another learning-induced, long-term change in sensory neurons appears to depend on a retrograde signal. It was reported by Dale et al. (1987) that repeated applications of 5-HT, similar to the treatment that produces long-term facilitation of in vitro synapses, produces long-term hyperexcitability of sensory neurons in vitro. In their study, Dale et al. used cultures of isolated sensory neurons as well as sensorimotor cocultures. They stated that the 5-HT treatment produced long-term hyperexcitability of isolated sensory neurons and of sensory neurons in synaptic contact with motor neurons. But a later study failed to find long-term hyperexcitability of isolated sensory neurons in culture after prolonged 5-HT treatment (Liao et al., 1999). Cai, $\mathrm{Li}$, and I, in agreement with Liao et al., have found that 5-HT does not produce persistent hyperexcitability of isolated sensory neurons; but repeated applications of 5-HT do result in long-term hyperexcitability of sensory neurons in sensorimotor cocultures (Cai et al., 2005). This result implies that the long-term enhancement of excitability in sensory neurons produced by 5-HT involves a retrograde signal.

I propose that all persistent, learning-induced changes in the sensory neuron depend on one or more, as-yet uniden- 
tified, retrograde signals. Further, I believe that these presynaptic changes are triggered by elevated postsynaptic $\mathrm{Ca}^{2+}$. Note that our evidence indicates that both homosynaptic LTD (Lin and Glanzman, 1996) and persistent heterosynaptic facilitation of the sensorimotor synapse ( $\mathrm{Li}$ et al., 2005) are blocked by the presence of BAPTA in the motor neuron (see also Murphy and Glanzman, 1996; Bao et al., 1998; Antonov et al., 2003). It is not yet known whether long-term (24-h) heterosynaptic facilitation, which involves significant, unambiguous morphological and molecular changes within the sensory neuron (Kandel, 2001), also requires a postsynaptic rise in $\mathrm{Ca}^{2+}$.

An interesting question is whether elevated postsynaptic $\mathrm{Ca}^{2+}$ is necessary for long-term presynaptic plasticity in the mammalian brain. NMDA receptor-dependent LTP of hippocampal synapses is induced postsynaptically and involves elevated postsynaptic $\mathrm{Ca}^{2+}$ (Malenka and Bear, 2004). Moreover, as pointed out above, presynaptic changes appear to contribute to the expression of this form of LTP (Antonova et al., 2001; Choi et al., 2003; Zakharenko et al., 2003; Powell, 2006). Given this situation, it is clear that these changes must be mediated by some, as yet unknown, retrograde signal. An apparent counterexample to the idea that long-term presynaptic changes depend on retrograde signals, however, is non-NMDA receptor-dependent LTP of the mossy fiber $\rightarrow$ CA3 pyramidal cell synapse. It has been claimed that this form of "nonassociative" LTP is both induced and expressed presynaptically (Zalutsky and Nicoll, 1990; Huang et al., 1994; Weisskopf et al., 1994). In particular, mossy fiber LTP appears to involve elevation of presynaptic cyclic AMP (cAMP). But there is also evidence that mossy fiber LTP, like NMDA receptor-dependent LTP, requires elevated postsynaptic $\mathrm{Ca}^{2+}$ for its induction (Williams and Johnston, 1989; Yeckel et al., 1999) (although see Mellor and Nicoll, 2001). In support of this idea, Contractor et al. (2002) have found that mossy fiber LTP involves trans-synaptic signaling mediated by an Eph receptor-ephrin pathway. According to their evidence, the LTP is triggered by a postsynaptic rise in intracellular $\mathrm{Ca}^{2+}$, which stimulates the binding of postsynaptic EphB receptors to presynaptic B-ephrins; the activation of presynaptic ephrins, in turn, causes stimulation of the cAMP/protein kinase A (PKA) pathway within the mossy fibers. It is attractive to consider the potential applicability of such a scheme to heterosynaptic facilitation of the Aplysia sensorimotor synapse, in which presynaptic cAMP and PKA also play prominent roles (Byrne and Kandel, 1996).

\section{Conclusion}

The present review has summarized recent data from studies of nonassociative learning in Aplysia. As the evidence from these studies shows, the contribution of postsynaptic mechanisms of plasticity to habituation and sensitization has until now been seriously underestimated. This work therefore reinforces the conclusions from studies of associative memory in Aplysia (reviewed in Roberts and Glanzman, 2003) that postsynaptic processes, particularly NMDA receptor-dependent LTP, are far more important than originally believed (Carew et al., 1984; Hawkins and Kandel, 1984; Byrne, 1987). The present situation is somewhat ironic, given the history of Aplysia research. Twenty years ago, work on Aplysia was at the forefront of the field of the cell biology of learning and memory. Today, attention has shifted to the mouse and rat, particularly in the area of associative memory. In part, this is because genetics and molecular biology are far more central to learning and memory research now than they were two decades ago, and Aplysia is not an advantageous organism for genetic studies of learning. But perhaps a more fundamental reason for the waning influence of Aplysia research, in particular, and invertebrate research, in general, in the field of learning and memory, has been the exaggeration by invertebrate researchers of the importance of exclusively presynaptic (or, in some cases, nonsynaptic) learning mechanisms. By comparison, the studies of learning-related plasticity in mammals have largely focused on postsynaptic mechanisms, such as NMDA receptor activation, postsynaptic kinase activity, and modulation of AMPA receptor trafficking (Lisman et al., 2002; Morris et al., 2003; Malenka and Bear, 2004). This difference in the intellectual focus of invertebrate and vertebrate researchers has led some to conclude, erroneously, that the simpler nervous systems of invertebrates do not express the postsynaptic forms of learning-related plasticity found in the more complex mammalian CNS. (See, for example, the striking contrast between the cellular mechanisms for learning in Aplysia and those in mammals given by Bear et al., 2007.) Fortunately, recent investigations of invertebrate learning and synaptic plasticity have begun to emphasize the critical contributions made by postsynaptic mechanisms (above, and Burrell and Sahley, 2004; Xia et al., 2005). Whether the belated recognition that common postsynaptic processes underlie invertebrate and vertebrate learning will restore invertebrate research to its former prominence within the field of learning and memory remains to be seen.

In summary, a comprehensive understanding of the neuronal and molecular changes that are triggered within the nervous system of Aplysia and other invertebrates during learning will require greater attention to postsynaptic mechanisms of plasticity, as well as the identification of the trans-synaptic signaling pathways whereby presynaptic and postsynaptic changes are coordinated. Finally, we will need to determine the contribution of plasticity at interneuronal sites to learning, a subject that has been relatively unexplored in Aplysia, (but see Trudeau and Castellucci, 
1993b; Tsau et al., 1994; Cleary et al., 1995; Frost and Kandel, 1995). A truly elephantine amount of work remains to be done.

\section{Acknowledgments}

The work from my laboratory discussed in this review was supported by National Institutes of Health Grants NS29563 and MH068543. I thank Dr. Daniel Fulton for comments on the manuscript.

\section{Literature Cited}

Antonov, I., I. Antonova, E. R. Kandel, and R. D. Hawkins. 2003. Activity-dependent presynaptic facilitation and Hebbian LTP are both required and interact during classical conditioning in Aplysia. Neuron 37: $135-147$.

Antonova, I., O. Arancio, A.-C. Trillat, H.-G. Wang, L. Zablow, H. Udo, E. R. Kandel, and R. D. Hawkins. 2001. Rapid increase in clusters of presynaptic proteins at onset of long-lasting potentiation. Science 294: 1547-1550.

Antzoulatos, E. G., L. J. Cleary, A. Eskin, D. A. Baxter, and J. H. Byrne. 2003. Desensitization of postsynaptic glutamate receptors contributes to high-frequency homosynaptic depression of Aplysia sensorimotor connections. Learn. Mem. 10: 309-313.

Armitage, B. A., and S. A. Siegelbaum. 1998. Presynaptic induction and expression of homosynaptic depression at Aplysia sensorimotor neuron synapses. J. Neurosci. 18: 8770-8779.

Bailey, C. H., and M. Chen. 1983. Morphological basis of long-term habituation and sensitization in Aplysia. Science 220: 91-93.

Bailey, C. H., and M. Chen. 1988a. Long-term memory in Aplysia modulates the total number of varicosities of single identified sensory neurons. Proc. Natl. Acad. Sci. USA 85: 2373-2377.

Bailey, C. H., and M. Chen. 1988b. Morphological basis of short-term habituation in Aplysia. J. Neurosci. 8: 2452-2459.

Bao, J. X., E. R. Kandel, and R. D. Hawkins. 1998. Involvement of presynaptic and postsynaptic mechanisms in a cellular analog of classical conditioning at Aplysia sensory-motor neuron synapses in isolated cell culture. J. Neurosci. 18: 458-466.

Bear, M. F., B. W. Connors, and M. A. Paradiso. 2007. Neuroscience: Exploring the Brain, 3rd ed. Lippincott, Williams \& Wilkins, Baltimore, MD.

Brunelli, M., V. Castellucci, and E. R. Kandel. 1976. Synaptic facilitation and behavioral sensitization in Aplysia: possible role of serotonin and cyclic AMP. Science 194: 1178-1181.

Burrell, B. D., and C. L. Sahley. 2004. Multiple forms of long-term potentiation and long-term depression converge on a single interneuron in the leech CNS. J. Neurosci. 24: 4011-4019.

Byrne, J. H. 1987. Cellular analysis of associative learning. Physiol. Rev. 67: 329-439.

Byrne, J. H., and E. R. Kandel. 1996. Presynaptic facilitation revisited: state and time dependence. J. Neurosci. 16: 425-435.

Cai, D., Q. Li, and D. L. Glanzman. 2005. Serotonin-induced longterm hyperexcitability of Aplysia sensory neurons in culture: dependence upon a retrograde signal from the postsynaptic motor neuron. Soc. Neurosci. Abstr. 31: 540.51. [Online].

Carew, T. J., and E. R. Kandel. 1973. Acquisition and retention of long-term habituation in Aplysia: correlation of behavioral and cellular processes. Science 182: 1158-1160.

Carew, T. J., H. M. Pinsker, and E. R. Kandel. 1972. Long-term habituation of a defensive withdrawal reflex in Aplysia. Science 175: 451-454.

Carew, T. J., R. D. Hawkins, T. W. Abrams, and E. R. Kandel. 1984.
A test of Hebb's postulate at identified synapses which mediate classical conditioning in Aplysia. J. Neurosci. 4: 1217-1224.

Castellucci, V. F., and E. R. Kandel. 1974. A quantal analysis of the synaptic depression underlying habituation of the gill-withdrawal reflex in Aplysia. Proc. Natl. Acad. Sci. USA 71: 5004-5008.

Castellucci, V., H. Pinsker, I. Kupfermann, and E. R. Kandel. 1970. Neuronal mechanisms of habituation and dishabituation of the gillwithdrawal reflex in Aplysia. Science 167: 1745-1748.

Chin, J., J. A. Burdohan, A. Eskin, and J. H. Byrne. 2002. Inhibitor of glutamate transport alters synaptic transmission at sensorimotor synapses in Aplysia. J. Neurophysiol. 87: 3165-3168.

Chitwood, R. A., Q. Li, and D. L. Glanzman. 2001. Serotonin facilitates AMPA-type responses in isolated siphon motor neurons of Aplysia in culture. J. Physiol. 534: 501-510.

Choi, S., J. Klingauf, and R. W. Tsien. 2003. Fusion pore modulation as a presynaptic mechanism contributing to expression of long-term potentiation. Philos. Trans. R. Soc. Lond. B Biol. Sci. 358: 695-705.

Cleary, L. J., J. H. Byrne, and W. N. Frost. 1995. Role of interneurons in defensive withdrawal reflexes in Aplysia. Learn. Mem. 2: 133-151.

Cohen, T. E., S. W. Kaplan, E. R. Kandel, and R. D. Hawkins. 1997. A simplified preparation for relating cellular events to behavior: mechanisms contributing to habituation, dishabituation, and sensitization of the Aplysia gill-withdrawal reflex. J. Neurosci. 17: 2886-2899.

Contractor, A., C. Rogers, C. Maron, M. Henkemeyer, G. T. Swanson, and S. F. Heinemann. 2002. Trans-synaptic Eph receptor-ephrin signaling in hippocampal mossy fiber LTP. Science 296: 1864-1869.

Dale, N., and E. R. Kandel. 1993. L-glutamate may be the fast excitatory transmitter of Aplysia sensory neurons. Proc. Natl. Acad. Sci. USA 90: 7163-7167.

Dale, N., E. R. Kandel, and S. Schacher. 1987. Serotonin produces long-term changes in the excitability of Aplysia sensory neurons in culture that depend on new protein synthesis. J. Neurosci. 7: 2232 2238 .

Dudek, S. M., and M. F. Bear. 1992. Homosynaptic long-term depression in area CA1 of the hippocampus and effects of $N$-methyl-Daspartate receptor blockade. Proc. Natl. Acad. Sci. USA 89: 43634367.

Ezzeddine, Y., and D. L. Glanzman. 2003. Prolonged habituation of the gill-withdrawal reflex in Aplysia depends on protein synthesis, protein phosphatase activity, and postsynaptic glutamate receptors. J. Neurosci. 23: 9585-9594.

Ezzeddine, Y., K. C. Pearce, and D. L. Glanzman. 2004. Long-term habituation of the gill-withdrawal reflex in Aplysia depends upon RNA synthesis and phosphatase 2B activity. Soc. Neurosci. Abstr. 30: 553.13. [Online].

Frost, L., S. W. Kaplan, T. E. Cohen, V. Henzi, E. R. Kandel, and R. D. Hawkins. 1997. A simplified preparation for relating cellular events to behavior: contribution of LE and unidentified siphon sensory neurons to mediation and habituation of the Aplysia gill- and siphonwithdrawal reflex. J. Neurosci. 17: 2900-2913.

Frost, W. N., and E. R. Kandel. 1995. Structure of the network mediating siphon-elicited siphon withdrawal in Aplysia. J. Neurophysiol. 73: 2413-2427.

Glanzman, D. L. 1994. Postsynaptic regulation of the development and long-term plasticity of Aplysia sensorimotor synapses in cell culture. J. Neurobiol. 25: 666-693.

Glanzman, D. L., S. L. Mackey, R. D. Hawkins, A. M. Dyke, P. E. Lloyd, and E. R. Kandel. 1989. Depletion of serotonin in the nervous system of Aplysia reduces the behavioral enhancement of gill withdrawal as well as the heterosynaptic facilitation produced by tail shock. J. Neurosci. 9: 4200-4213.

Glanzman, D. L., E. R. Kandel, and S. Schacher. 1990. Targetdependent structural changes accompanying long-term synaptic facilitation in Aplysia neurons. Science 249: 799-802. 
Hawkins, R. D., and E. R. Kandel. 1984. Is there a cell biological alphabet for simple forms of learning? Psychol. Rev. 91: 375-391.

Hawkins, R. D., T. W. Abrams, T. J. Carew, and E. R. Kandel. 1983. A cellular mechanism of classical conditioning in Aplysia: activity-dependent amplification of presynaptic facilitation. Science 219: 400-405.

Hawkins, R. D., T. E. Cohen, W. Greene, and E. R. Kandel. 1998. Relationships between dishabituation, sensitization, and inhibition of the gill- and siphon-withdrawal reflex in Aplysia californica: effects of response measure, test time, and training stimulus. Behav. Neurosci. 112: $24-38$.

Hochner, B., M. Klein, S. Schacher, and E. R. Kandel. $1986 . \quad$ Additional component in the cellular mechanism of presynaptic facilitation contributes to behavioral dishabituation in Aplysia, Proc. Natl. Acad. Sci. USA 83: 8794-8798.

Huang, Y.-Y., X.-C. Li, and E. R. Kandel. 1994. cAMP contributes to mossy fiber LTP by initiating both a covalently mediated early phase and macromolecular synthesis-dependent late phase. Cell 79: 69-79.

Kandel, E. R. 2001. The molecular biology of memory storage: a dialogue between genes and synapses. Science 294: 1030-1038.

Kandel, E. R., M. Brunelli, J. Byrne, and V. Castellucci. 1975. A common presynaptic locus for the synaptic changes underlying shortterm habituation and sensitization of the gill-withdrawal reflex in Aplysia. Cold Spring Harbor Symp. Quant. Biol. 40: 465-482.

Kirkwood, A., and M. F. Bear. 1994. Homosynaptic long-term depression in the visual cortex. J. Neurosci. 14: 3404-3412.

Levenson, J., D. M. Sherry, L. Dryer, J. Chin, J. H. Byrne, and A. Eskin. 2000. Localization of glutamate and glutamate transporters in the sensory neurons of Aplysia. J. Comp. Neurol. 423: 121-131.

Li, Q., A. C. Roberts, and D. L. Glanzman. 2005. Synaptic facilitation and behavioral dishabituation in Aplysia: dependence upon release of $\mathrm{Ca}^{2+}$ from postsynaptic intracellular stores, postsynaptic exocytosis and modulation of postsynaptic AMPA receptor efficacy. J. Neurosci. 25: 5623-5637.

Liao, X., C. G. Brou, and E. T. Walters. 1999. Limited contributions of serotonin to long-term hyperexcitability of Aplysia sensory neurons. J. Neurophysiol. 82: 3223-3235.

Lin, X. Y., and D. L. Glanzman. 1994a. Hebbian induction of long-term potentiation of Aplysia sensorimotor synapses: partial requirement for activation of an NMDA-related receptor. Proc. Biol. Sci. 255: 215-221.

Lin, X. Y., and D. L. Glanzman. 1994b. Long-term potentiation of Aplysia sensorimotor synapses in cell culture: regulation by postsynaptic voltage. Proc. Biol. Sci. 255: 113-118.

Lin, X. Y., and D. L. Glanzman. 1996. Long-term depression of Aplysia sensorimotor synapses in cell culture: inductive role of a rise in postsynaptic calcium. J. Neurophysiol. 76: 2111-2114.

Lisman, J., H. Schulman, and H. Cline. 2002. The molecular basis of CaMKII function in synaptic and behavioral memory. Nat. Rev. Neurosci. 3: 175-190.

Lledo, P. M., X. Zhang, T. C. Seudhof, R. C. Malenka, and R. A. Nicoll. 1998. Postsynaptic membrane fusion and long-term potentiation. Science 279: 399-403.

Mackey, S. L., E. R. Kandel, and R. D. Hawkins. 1989. Identified serotonergic neurons LCB1 and RCB1 in the cerebral ganglia of Aplysia produce presynaptic facilitation of siphon sensory neurons. J. Neurosci. 9: 4227-4235.

Malinow, R., and R. C. Malenka. 2002. AMPA receptor trafficking and synaptic plasticity. Аппи. Rev. Neurosci. 25: 103-126.

Malenka, R. C., and M. F. Bear. 2004. LTP and LTD: an embarrassment of riches. Neuron 44: 5-21.

Malenka, R. C., and R. A. Nicoll. 1999. Long-term potentiation-a decade of progress? Science 285: 1870-1874.

Marcus, E. A., T. G. Nolen, C. H. Rankin, and T. J. Carew. 1988. Behavioral dissociation of dishabituation, sensitization, and inhibition in Aplysia. Science 241: 210-213.
Marinesco, S., and T. J. Carew. 2002. Serotonin release evoked by tail nerve stimulation in the CNS of Aplysia: characterization and relationship to heterosynaptic plasticity. J. Neurosci. 22: 2299-2312.

Mellor, J., and R. A. Nicoll. 2001. Hippocampal mossy fiber LTP is independent of postsynaptic calcium. Nat. Neurosci. 4: 125-126.

Montarolo, P. G., P. Goelet, V. F. Castellucci, J. Morgan, E. R. Kandel, and S. Schacher. 1986. A critical period for macromolecular synthesis in long-term heterosynaptic facilitation in Aplysia. Science 234: $1249-1254$.

Morris, R. G., E. I. Moser, G. Riedel, S. J. Martin, J. Sandin, M. Day, and C. O'Carroll. 2003. Elements of a neurobiological theory of the hippocampus: the role of activity-dependent synaptic plasticity in memory. Philos. Trans. R. Soc. Lond. B Biol. Sci. 358: 773-786.

Mulkey, R. M., and R. C. Malenka. 1992. Mechanisms underlying induction of homosynaptic long-term depression in area CA1 of the hippocampus. Neuron 9: 967-975.

Mulkey, R. M., C. E. Herron, and R. C. Malenka. 1993. An essential role for protein phosphatases in hippocampal long-term depression. Science 261: 1051-1055.

Mulkey, R. M., S. Endo, S. Shenolikar, and R. C. Malenka. 1994. Involvement of a calcineurin/inhibitor-1 phosphatase cascade in hippocampal long-term depression. Nature 369: 486-488.

Murphy, G. G., and D. L. Glanzman. 1996. Enhancement of sensorimotor connections by conditioning-related stimulation in Aplysia depends upon postsynaptic $\mathrm{Ca}^{2+}$. Proc. Natl. Acad. Sci. USA 93: 9931-9936.

Murphy, G. G., and D. L. Glanzman. 1997. Mediation of classical conditioning in Aplysia californica by LTP of sensorimotor synapses. Science 278: 467-471.

Murphy, G. G., and D. L. Glanzman. 1999. Cellular analog of differential classical conditioning in Aplysia: disruption by the NMDA receptor-antagonist DL-2-amino-5-phosphonovalerate. J. Neurosci. 19: 10595-10602.

Nicoll, R. A. 2003. Expression mechanisms underlying long-term potentiation: a postsynaptic view. Philos. Trans. R. Soc. Lond. B Biol. Sci. 358: 721-726.

Pinsker, H., I. Kupfermann, V. Castellucci, and E. Kandel. 1970. Habituation and dishabituation of the gill-withdrawal reflex in Aplysia. Science 167: 1740-1742.

Powell, C. M. 2006. Gene targeting of presynaptic proteins in synaptic plasticity and memory: across the great divide. Neurobiol. Learn. Mem. 85: $2-15$.

Rankin, C. H., and T. J. Carew. 1988. Dishabituation and sensitization emerge as separate processes during development in Aplysia. J. Neurosci. 8: 197-211.

Rankin, C. H., and S. R. Wicks. 2000. Mutations of the Caenorhabditis elegans brain-specific inorganic phosphate transporter eat-4 affect habituation of the tap-withdrawal response without affecting the response itself. J. Neurosci. 20: 4337-4344.

Roberts, A. C., and D. L. Glanzman. 2003. Learning in Aplysia: looking at synaptic plasticity from both sides. Trends Neurosci. 26: 662-670.

Rose, J. K., and C. H. Rankin. 2001. Analyses of habituation in Caenorhabditis elegans. Learn. Mem. 8: 63-69.

Rose, J. K., K. R. Kaun, S. H. Chen, and C. H. Rankin. 2003. GLR-1, a non-NMDA glutamate receptor homolog, is critical for long-term memory in Caenorhabditis elegans. J. Neurosci. 23: 9595-9599.

Rumpel, S., J. LeDoux, A. Zador, and R. Malinow. 2005. Postsynaptic receptor trafficking underlying a form of associative learning. Science 308: $83-88$

Trudeau, L. E., and V. F. Castellucci. 1993a. Excitatory amino acid neurotransmission at sensory-motor and interneuronal synapses of Aplysia californica. J. Neurophysiol. 70: 1221-1230.

Trudeau, L. E., and V. F. Castellucci. 1993b. Functional uncoupling of inhibitory interneurons plays an important role in short-term sensitization of Aplysia gill and siphon withdrawal reflex. J. Neurosci. 13: 2126-2135. 
Tsau, Y., J. Y. Wu, H. P. Hopp, L. B. Cohen, D. Schiminovich, and C. X. Falk. 1994. Distributed aspects of the response to siphon touch in Aplysia: spread of stimulus information and cross-correlation analysis. J. Neurosci. 14: 4167-4184.

Walters, E. T., and J. H. Byrne. 1983. Associative conditioning of single sensory neurons suggests a cellular mechanism for learning. Science 219: 405-408.

Weisskopf, M. G., P. E. Castillo, R. A. Zalutsky, and R. A. Nicoll. 1994. Mediation of hippocampal mossy fiber long-term potentiation by cyclic AMP. Science 265: 1878-1882.

Williams, S., and D. Johnston. 1989. Long-term potentiation of hippocampal mossy fiber synapses is blocked by postsynaptic injection of calcium chelators. Neuron 3: 583-588.

Xia, S., T. Miyashita, T. F. Fu, W. Y. Lin, C. L. Wu, L. Pyzocha, I. R. Lin,
M. Saitoe, T. Tully, and A. S. Chiang. 2005. NMDA receptors mediate olfactory learning and memory in Drosophila. Curr. Biol. 15: 603-615.

Yeckel, M. F., A. Kapur, and D. Johnston. 1999. Multiple forms of LTP in hippocampal CA3 neurons use a common postsynaptic mechanism. Nat. Neurosci. 2: 625-633.

Zakharenko, S. S., S. L. Patterson, I. Dragatsis, S. O. Zeitlin, S. A. Siegelbaum, E. R. Kandel, and A. Morozov. 2003. Presynaptic BDNF required for a presynaptic but not postsynaptic component of LTP at hippocampal CA1-CA3 synapses. Neuron 39: 975-990.

Zalutsky, R. A., and R. A. Nicoll. 1990. Comparison of two forms of long-term potentiation in single hippocampal neurons. Science $\mathbf{2 4 8}$ : 1619-1624. 